University of Warwick institutional repository: http://go.warwick.ac.uk/wrap This paper is made available online in accordance with publisher policies. Please scroll down to view the document itself. Please refer to the repository record for this item and our policy information available from the repository home page for further information.

To see the final version of this paper please visit the publisher's website. Access to the published version may require a subscription.

Author(s): M. POLLICOTT, R. SHARP, S. TUNCEL and P. WALTERS Article Title: The mathematical research of William Parry FRS Year of publication: 2008 Link to published version: http://dx.doi.org/ 10.1017/S0143385708000102

Publisher statement: None 


\title{
The mathematical research of William Parry FRS
}

\author{
M. POLLICOTT $\dagger$, R. SHARP $\ddagger$, S. TUNCEL $\S$ and P. WALTERS $\dagger$ \\ $\dagger$ Department of Mathematics, University of Warwick, Coventry CV4 7AL, UK \\ (e-mail: m.pollicott@warwick.ac.uk) \\ $\ddagger$ School of Mathematics, University of Manchester, Oxford Road, \\ Manchester M13 9PL, UK \\ $\S$ Department of Mathematics, University of Washington, Box 354350 Seattle, \\ WA 98195-4350, USA
}

(Received 25 February 2008 and accepted in revised form 25 February 2008)

Abstract. In this article we survey the mathematical research of the late William (Bill) Parry, FRS.

\section{Introduction}

In this brief survey we attempt to give an account of at least part of Bill Parry's wide ranging mathematical work during his long and distinguished career. Beyond his research publications, Bill has also left a lasting legacy to the scientific community through his books, and his role in both establishing the journal Ergodic Theory and Dynamical Systems and a leading international school in ergodic theory at Warwick University where he worked from 1968 until his retirement in 1999. During his career he had $20 \mathrm{PhD}$ students, the majority of whom were inspired to continue in academic careers.

Bill Parry was born on 3 July 1934 in Tile Hill, Coventry, UK and died on 20th August 2006 in Coventry, from cancer exacerbated by an infection. His career as a research mathematician started as a PhD student (1956-1960) at Imperial College London, under the supervision of Yael Dowker. It was there that Bill began his study of ergodic theory. He had previously studied for an MSc at Liverpool, where he had developed his background in measure theory. His first published paper in 1960 was typical of a recurring theme throughout his career, namely the interaction between ergodic theory and number theory. This was on $\beta$-expansions, whose study had been initiated by the Hungarian mathematician Alfred Renyi.

Over his long career he made important contributions to a number of topics, within both ergodic theory and related areas. Of these, perhaps the most influential was the original construction of the measure of maximal entropy for topological Markov chains, subsequently widely known as the Parry measure. In addition, he also made fundamental contributions to the theory of affine transformations and nilflows, entropy theory, the classification of subshifts of finite type and the theory of hyperbolic systems, their zeta 
functions and cocycles. Typically, his papers were characterized by their brevity, clarity and insight, and were always the product of hard work and many revisions.

More details of Bill Parry's life and career appear in the obituary for the Royal Society of London (written by Mary Rees).

\section{Early work}

2.1. $\quad \beta$-expansions and interval maps. In Bill Parry's first paper [1], based on his $\mathrm{PhD}$ thesis, he studied $\beta$-expansions of real numbers. More precisely, let $\beta$ be an arbitrary positive number greater than 1 , which is not an integer. Every real number $0<x<1$ has a $\beta$-expansion of the form

$$
x=\sum_{k=1}^{\infty} \frac{\varepsilon_{k}}{\beta^{k}}
$$

where the coefficients $\varepsilon_{k}=\varepsilon_{k}(x)$ take the values $0,1, \ldots,[\beta]$. Bill Parry gave sufficient conditions on a sequence $\left(\varepsilon_{k}\right)$ of integers $\left(0 \leq \varepsilon_{k} \leq[\beta], k=1,2, \ldots\right)$ in order that it should arise as a sequence of digits of a $\beta$-expansion. Those numbers for which the $\beta$-expansion is finite (i.e. there exists $N$ such that $\varepsilon_{n}(1)=0$, for all $n \geq N$ ) were called simple $\beta$-numbers and he showed that the set of such numbers is everywhere dense in $(1,+\infty)$. He also showed that the set

$$
V_{\beta}:=\overline{\left\{\varepsilon_{k}(x) \mid x \in[0,1)\right\}} \subset\{0, \ldots,[\beta]\}^{\mathbb{Z}^{+}}
$$

is a subshift of finite type if and only if the $\beta$ is simple.

It was shown by Rényi in $1957[\mathbf{1 1 8}]$ that the transformation $T:[0,1) \rightarrow[0,1)$ defined by $T(x)=\{\beta x\}:=\beta x-[\beta x]$ (the fractional part of $\beta x$ ) has a unique $T$-invariant probability measure $v_{\beta}$ equivalent to Lebesgue measure. Moreover, the measure $v_{\beta}$ is ergodic. Bill Parry showed that the Radon-Nikodym derivative has the particular form

$$
\frac{d v_{\beta}}{d x}(x)=C \sum_{n: x<T^{n}(1)} \frac{1}{\beta^{n}},
$$

for some normalization constant $C>0$ (a result that was also discovered independently by A. O. Gelfond). Four years later he revisited these ideas in the study of the more general $f$-expansions of real numbers, in particular linear mod 1 transformations of the form $T(x)=\{\beta x+\alpha\}$, where $\beta \geq 1$ and $0 \leq \alpha \leq 1[8]$.

Bill Parry's second published paper [2] was written after he moved to his first job at the University of Birmingham. It concerned a transformation, introduced by Henry Daniels who was Professor of Statistics at Birmingham, which was later called the ParryDaniels map. Let $\Delta=\left\{x=\left(x_{1}, \ldots, x_{n}\right) \in \mathbb{R}^{n} \mid 0 \leq x_{i} \leq 1,0 \leq i \leq n, \sum_{i=1}^{n} x_{i}=1\right\}$ be the simplex in $\mathbb{R}^{n}$. To almost every point $x \in \Delta$ there is a unique permutation $\pi$ with $x_{\pi(1)}<x_{\pi(2)}<\cdots<x_{\pi(n)}$ and we can define the map $T: \Delta \rightarrow \Delta$ by

$$
T\left(x_{1}, \ldots, x_{n}\right)=\left(\frac{x_{\pi(1)}}{x_{\pi(n)}}, \frac{x_{\pi(2)}-x_{\pi(1)}}{x_{\pi(n)}}, \ldots, \frac{x_{\pi(n)}-x_{\pi(n-1)}}{x_{\pi(n)}}\right) .
$$

Daniels had found the density function for a $T$-invariant $\sigma$-finite measure on $\Delta$ and Bill Parry showed in the case where $n=2$ that $T$ is ergodic by relating $T$ to the Gauss map $x \mapsto\{1 / x\}$ used to study continued fractions. Many years later higher dimensional cases were studied by Nogueira [107] and Schweiger [113]. 
In 1966, Bill Parry published what proved to be a particularly far-sighted paper on the topological classification of interval maps. More precisely, he gave conditions under which a strongly transitive piecewise monotone transformation $T: I \rightarrow I$ of the unit interval onto itself is conjugate to a piecewise linear transformation. Let $\left\{\left(a_{i}, a_{i+1}\right)\right\}$ denote the open intervals of monotonicity and assume that either:

(1) the sets $T\left(a_{i}, a_{i+1}\right) \cap T\left(a_{j}, a_{j+1}\right) \neq \emptyset$ for two distinct intervals $\left(a_{i}, a_{i+1}\right)$ and $\left(a_{j}, a_{j+1}\right)$; or

(2) the images $T\left(a_{i}, a_{i+1}\right)$ are pairwise disjoint and $T$ has no periodic points;

then $T$ is topologically conjugate to a piecewise linear map $S: I \rightarrow I$ [14]. In particular, the classical Poincaré-Denjoy theorem about homeomorphisms of the unit circle becomes a corollary. The method of proof uses subshifts of finite type, and anticipates later work on symbolic dynamics.

In later years the study of interval maps became the focus of considerable activity.

2.2. Infinite measure spaces. In 1962-1963, Bill Parry spent the academic year at Yale University, and this gave him the opportunity to explore new ideas and to interact with other mathematicians (including S. Kakutani, and his students Roy Adler and Joe Auslander, who remained lifelong friends of Bill). During this period, he collaborated with Kakutani on problems connected with the properties of infinite $\sigma$-finite measures. For example, if a transformation $T: X \rightarrow X$ preserves a finite measure, then it is well known from work of Halmos that $T^{(k)}=T \times \cdots \times T$, the direct product of $k$ copies of $T$, is ergodic for any $k \geq 2$ if and only if $T^{(2)}$ is ergodic. However, Kakutani and Parry showed that for infinite measures the situation was very different. They gave, for each positive integer $k$, an example of a transformation $T$ which preserves a $\sigma$-finite infinite measure and such that $T^{(k)}$ is ergodic but $T^{(k+1)}$ is not [3].

Ergodicity and the Kolmogorov property are invariants for isomorphism, even in the $\sigma$-finite case. However, although it is known that for finite measures ergodicity is also a spectral invariant, Bill Parry showed that the situation can be entirely different in the $\sigma$-finite case, and that an ergodic transformation can be unitarily equivalent to a non-ergodic one.

At about the same time, Bill also developed versions of Hurewicz's ergodic theorem and McMillan's ergodic theorem without the hypothesis of the existence of an invariant probability [7]. Interestingly, Hurewicz was Bill Parry's 'mathematical grandfather'.

2.3. Subshifts and the Parry measure. In 1964, Bill Parry published an article entitled 'Intrinsic Markov chains', which was to prove one of his most influential papers [5]. In modern language, this involved the study of subshifts of finite type and showed the existence of a unique measure of maximal entropy for aperiodic subshifts of finite type. More precisely, let $A$ be a $k \times k$ matrix with entries either zero or one which is aperiodic (i.e. $A^{n}$ has all entries positive for some $n \geq 1$ ) and let

$$
\Sigma_{A}=\left\{\left(x_{n}\right) \in \prod_{k=-\infty}^{\infty}\{1, \ldots, k\} \mid A\left(x_{n}, x_{n+1}\right)=1, n \in \mathbb{Z}\right\}
$$


and $\sigma: \Sigma_{A} \rightarrow \Sigma_{A}$ be the shift map. The topological entropy $h(\sigma)$ is equal to $\log \lambda$, where $\lambda>1$ is the maximal eigenvalue of $A$ guaranteed by the Perron-Frobenius theorem. If $v$ is the positive right eigenvector for $A$ corresponding to the eigenvalue $\lambda$ then one associates a stochastic matrix $P$ by $P(i, j)=A(i, j) v_{j} /\left(\lambda v_{i}\right)$. If we let $p$ be the probability vector with $p P=p$ then one can define the $\sigma$-invariant Markov measure $m_{P}$ by

$$
m_{P}\left[x_{0}, \ldots, x_{n-1}\right]=p_{x_{0}} P\left(x_{0}, x_{1}\right) \cdots P\left(x_{n-2}, x_{n-1}\right) .
$$

Bill Parry showed that $m_{P}$ is the unique measure for which the measure-theoretic entropy $h_{m_{P}}(\sigma)$ is equal to the topological entropy $h(\sigma)$, all other $\sigma$-invariant probability measures having strictly smaller entropy. This measure of maximal entropy has subsequently become known as the Parry measure.

This original variational principle for entropy anticipated many of the developments in thermodynamic formalism a decade later, including the variational principle for pressure (proved for subshifts of finite type by David Ruelle [110], and for general continuous maps on compact spaces by Walters [116]).

2.4. Entropy and generators. At the end of the 1950s, A. Kolmogorov and Ya. G. Sinai introduced entropy as an invariant for isomorphism of measure-preserving transformations of probability spaces. Let $(X, \mathcal{B}, m)$ be a Lebesgue space and $Z$ the set of all countable measurable partitions $\xi$ of $X$ whose entropy is finite [109].

If $T: X \rightarrow X$ is an invertible measure-preserving transformation, then a partition $\xi$ is called a generator if every $B \in \mathcal{B}$ is equal almost everywhere to a set in the smallest $\sigma$-algebra containing all sets in the partitions $T^{-j} \xi, j \in \mathbb{Z}$, and is called a strong generator if every $B \in \mathcal{B}$ is equal almost everywhere to a set in the smallest $\sigma$-algebra containing all sets in the partitions $T^{-j} \xi$, for $j \geq 0$. Subsequently, V. A. Rohlin proved that if $T$ is aperiodic and has finite entropy then $T$ possesses a countable generator with finite entropy. Bill Parry extended this result for transformations with infinite entropy and even proved that there is a countable strong generator $[\mathbf{1 9}, \mathbf{2 0}]$. These results were also proved independently by Rohlin [119].

Bill Parry gave a series of lectures on these, and related, results at Yale University in 1966, which became the basis of his elegant book Entropy and Generators in Ergodic Theory [26]. Soon after, Ornstein proved that entropy is a complete isomorphism invariant for Bernoulli transformations [108].

2.5. Affine maps, nilflows and G-extensions. During his visit to Yale in 1962-1963, Bill Parry worked with Frank Hahn on topological discrete quasi-spectra which lead to the study of affine transformations on compact abelian groups. He continued this work in Birmingham with Howard Hoare. After learning of the importance of nilmanifolds from Smale's 1967 survey paper [115], Bill Parry studied ergodicity and minimality conditions for affine transformations of nilmanifolds and for nilflows on nilmanifolds. This, together with the work of Furstenberg, motivated his study of group extensions.

Bill Parry also proved one of the precursors of modern rigidity results. Assume $T_{i}$ : $X_{i} \rightarrow X_{i}$ are ergodic unipotent affine transformations of nilmanifolds $X_{i}$ onto themselves 
$(i=1,2)$; then we say that $\left(X_{1}, T_{1}\right)$ and $\left(X_{2}, T_{2}\right)$ are algebraically conjugate if there is a one-to-one affine transformation $\pi$ of $X_{1}$ onto $X_{2}$ such that $\pi \circ T_{1}=T_{2} \circ \pi$. He showed that if $\pi$ is a measurable map of $X_{1}$ onto almost all of $X_{2}$ such that $\pi\left(T_{1}(x)\right)=T_{2}(\pi(x))$ almost everywhere, then there exists an affine transformation $\pi^{\prime}$ of $X_{1}$ onto $X_{2}$ such that $\pi=\pi^{\prime}$ almost everywhere and $\pi^{\prime} \circ T_{1}=T_{2} \circ \pi^{\prime}$. This anticipated the later work of Ratner, Margulis and others on more general unipotent flows [106].

A dynamical system $\widehat{T}: \widehat{X} \rightarrow \widehat{X}$ commuting with the action of a compact group $G$ induces a factor $T$ on the space $X=\widehat{X} / G$ of orbits of the action, and $\widehat{T}$ is called a $G$-extension of the system $T$. If $G$ acts freely then any $G$-extension of $T$ has the form $\widehat{S}^{n}(\hat{x})=R_{\theta(x, n)} \widehat{T}^{n}(\hat{x})$, where $R_{g}(x)=g x$, for some fixed $G$-extension $\widehat{T}$. The $G$-valued function $\theta: X \times \mathbb{Z} \rightarrow G$ is a cocycle satisfying $g \theta(x, n)=\theta(g x, n)$, for $g \in G$ and $\theta(x, n+m)=\theta\left(T^{n} x, m\right) \theta(x, n)$. We say that extensions $\widehat{S}_{1}$ and $\widehat{S}_{2}$ are isomorphic if the cocycles are cohomologous, i.e. there exists a measurable function $\phi(x)$ such that $\theta_{1}(x, n)=\phi\left(T^{n} x\right) \theta_{2}(x, n) \phi(x)$. Bill Parry studied topological properties of $G$-extensions when $G$ is abelian and obtained a structure theorem for the class of minimal transformation with generalized discrete spectrum by representing such a map as an inverse limit of group extensions [25].

Roger Jones and Bill Parry proved that cocycles with values in abelian compact groups, homologous to the trivial cocycle, form a set of the first category in the group of cocycles (with uniform or $L^{1}$ topology) [35]. In particular, it follows that abelian $G$-extensions typically inherit the dynamical properties of the base.

Some of the ideas from $G$-extensions have echoes in his later work on skew products over hyperbolic systems.

2.6. Lebesgue spaces and Jacobians. Given a Lebesgue space $(X, \mathcal{B}, m)$, Bill Parry and Peter Walters studied isomorphism invariants for non-invertible measure-preserving transformations $T: X \rightarrow X$. These included the index function $i_{T}(x)$, the sigma algebra $\beta(T)$ and the jacobian $j_{T}(x)$, defined by $j_{T}(x)=1 / m\left(x \mid T^{-1} T x\right)$. They showed that these invariants are not sufficient to classify endomorphisms. More precisely, for two endomorphisms $S$ and $T$ to be isomorphic one needs the two sequences of $\sigma$-algebras $\left\{T^{-n} B\right\}$ and $\left\{S^{-n} B\right\}$ to be isomorphic. Parry and Walters showed that there are nonisomorphic exact endomorphisms $S$ and $T$ with $S^{2}=T^{2}, S^{-n} \mathcal{B}=T^{-n} \mathcal{B}$ for all $n \geq 0$, $j_{S} \equiv j_{T}$ and $\beta(S)=\beta(T)[\mathbf{1 1 6}]$.

More generally, the jacobian has proved a useful device for establishing the regularity of conjugacies between expanding one-dimensional maps, for example in work of Mike Shub and Dennis Sullivan on rigidity of conjugacies for expanding maps of the circle [114].

\section{The middle period}

There is no corresponding theory in topological dynamics to Ornstein's for Bernoulli measure-preserving transformations. For this reason variants of the conjugacy problem (for both the topological conjugacy problem and related conjugacy problems) have been considered in the particular setting of irreducible subshifts of finite type. Much of Bill Parry's work in this period (of nearly 15 years starting around 1970) concerned 
classifications of shifts of finite type and of Markov chains. It was motivated by three breakthroughs that took place around 1970 .

(1) The result of Don Ornstein and Nat Friedman on the measure-theoretic isomorphism of Markov chains [102].

(2) The work of Roy Adler and Benjy Weiss on Markov partitions for toral automorphisms [120], later generalized by Sinai and Rufus Bowen to Axiom A diffeomorphisms [99], which led Adler, Parry and others to ask about common extensions of subshifts of finite type.

(3) The work of Bob Williams, which we briefly describe below.

In the topological setting, Williams showed that two subshifts of finite type $\left(\Sigma_{A}, \sigma\right)$ and $\left(\Sigma_{B}, \sigma\right)$ are topologically conjugate if and only if $A$ and $B$ are strong shift equivalent, i.e. there are sequences of matrices $R_{1}, \ldots, R_{k}$ and $S_{1}, \ldots, S_{k}$ such that $R_{i} S_{i}=S_{i} R_{i}\left(R_{i}, S_{i}\right.$ not necessarily square matrices) with $A=R_{1} S_{1}$ and $B=S_{k} R_{k}$ [117]. Furthermore, he introduced the concept of shift equivalence, i.e. there exist matrices $U, V$ and an integer $m$ such that $U A=B U, A V=V B, U V=B^{m}, V U=A^{m}$. However, he also produced an erroneous proof that shift equivalence of $A$ and $B$ implies topological conjugacy of the associated subshifts of finite type. This claim subsequently became known as the Williams conjecture. When Bill Parry discovered the mistake in Williams's paper, he was motivated (as were a number of others) to work on this conjecture. Indeed, he thought about this problem, on and off, for many years, although it was Kim and Roush who eventually produced a counter-example to the Williams's conjecture in 1999 [103].

3.1. Shift equivalence of Markov measures. Given a subshift of finite type $\sigma: \Sigma_{A} \rightarrow$ $\Sigma_{A}$, let $m_{P}$ denote the Markov measure associated to a stochastic matrix $P$. Subshifts associated with stochastic matrices $P$ and $Q$ are said to be block isomorphic if there exists a topological conjugacy $\varphi$ such that $m_{Q}=m_{P} \varphi^{-1}$. One says that they are strong shift equivalent if there exist stochastic matrices $U_{1}, \ldots, U_{n}, V_{1}, \ldots, V_{n}$ such that $P=U_{1} V_{1}$, $V_{1} U_{1}=U_{2} V_{2}, \ldots, V_{n} U_{n}=Q$. Given a real number $t$, let $P^{t}$ denote the matrix of entries $P(i, j)^{t}$ (with the convention $0^{0}=0$ ). One says that they are shift equivalent if there exist $n$, called the lag, and matrices $V(t), U(t)$ consisting of entries which are non-negative integral combinations of exponential functions $e^{a t}, a \in \mathbb{R}$, such that $V(t) P^{t}=Q^{t} V(t)$, $P^{t} U(t)=U(t) Q^{t}, U(t) V(t)=P^{t} \cdots P^{t}$ (matrix multiplication $n$ times), $V(t) U(t)=$ $Q^{t} \cdots Q^{t}$ ( $n$ times). One says that they are adapted shift equivalent if $P_{(l)}$ is shift equivalent to $Q_{(l)}$ with lag $l$, where $P_{(l)}$ and $Q_{(l)}$ are the stochastic matrices associated with the $l$-block presentations of $P$ and $Q$. For $P^{t}$ we define the beta function $\beta(t)$ as its maximum eigenvalue for each $t \in \mathbb{R}$ and the zeta function by $\zeta(s)=\operatorname{det}\left(I-P^{s}\right)^{-1}$. Bill Parry and Selim Tuncel showed the following chain of implications [58]: block isomorphism $\Leftrightarrow$ strong shift equivalence $\Leftrightarrow$ adapted shift equivalence $\Rightarrow$ shift equivalence $\Rightarrow$ identical zeta functions $\Rightarrow$ identical beta functions $\Rightarrow$ identical topological entropies and measure-theoretic entropies.

3.2. Finite equivalence. Another notion of equivalence of subshifts of finite type is finite equivalence. In particular, two transitive subshifts of finite type are said to be finitely 
equivalent if they have a common finite-to-one continuous extension by a subshift of finite type.

Bill Parry used the decomposition of a non-negative irreducible matrix into the product of a division matrix and an amalgamation matrix, in the context of finite extensions, to show that topological entropy is a complete invariant for finite equivalence of subshifts of finite type. This was the first complete classification result for subshifts of finite type [46].

This construction was refined two years later by Roy Adler and Brian Marcus to achieve a complete classification (by entropy and period) in the case of almost topological conjugacy [121]. Bill Parry's classification result concerned common extensions by finiteto-one continuous maps whereas the Adler-Marcus classification result was by continuous finite extensions that are one-to-one almost everywhere. In 1990, Jonathan Ashley proved an elegant theorem that showed that (periodicity allowing) a continuous finite-to-one factor map can be replaced by a one-to-one almost everywhere map [97].

The work of Don Ornstein led Bill Parry and others to ask about more effective classifications (by maps that would be constructible in finite time, or by conditions that could be checked via an algorithm) for Markov chains. Bill Parry wrote a number of papers, some in collaboration, on invariants, and he sought to generalize Williams's theory and other results from subshifts of finite type (with the Parry measure) to arbitrary Markov chains. Bill Parry and Selim Tuncel found a suitable setting [56, 59] for this. After initially using exponentials, they subsequently realized that polynomials in several variables provided an equivalent (but more useful) formulation. The use of polynomials allowed Marcus and Tuncel to discover structures that established the main conjecture of [56] (i.e. that the beta function is a complete invariant for finite equivalence) in the case of polynomial beta functions, while giving a counter-example in the general case of Markov chains [104].

\subsection{Finitary isomorphisms. A measure-preserving isomorphism $\phi: \Sigma_{A} \rightarrow \Sigma_{B}$} between two subshifts of finite type takes the form $(\phi(x))_{n}=\phi_{0}\left(\sigma^{n} x\right)$ where $\phi_{0}$ is a map from $\Sigma_{A}$ to the symbol space of $\Sigma_{B}$. An isomorphism $\phi$ between two subshifts of finite type is said to be finitary provided that for almost all $x \in \Sigma_{A}$ there exists a positive integer $N_{x}$ such that the $\phi_{0}(x)$ and $\phi_{0}\left(x^{\prime}\right)$ agree for almost all $x^{\prime} \in \Sigma_{A}$ with $x_{i}=x_{i}^{\prime}$ for $|i| \leq N_{x}$ ( $x_{i}$ denotes the $i$ th coordinate of the point $x \in \Sigma_{A}$ ), and similarly for $\phi^{-1}$.

Mike Keane and Meir Smorodinsky were motivated by the work of Ornstein to study finitary isomorphism, culminating in their result that entropy and period are complete invariants in this case as well [122]. Bill Parry then asked about finitary isomorphisms with finite expected code length, and used invariants he had employed earlier to show that this situation was very different [51]. This motivated a number of researchers (Wolfgang Krieger, Klaus Schmidt, Selim Tuncel, in addition to Bill Parry) to work on finitary isomorphisms with finite expected code length, and the closely related hyperbolic isomorphisms.

The information cocycle of a shift $\sigma$ is defined as $I_{\sigma}=I\left(\alpha \mid \bigvee_{i=1}^{\infty} \sigma^{-i} \alpha\right)$, where $\alpha=\left\{A_{1}, \ldots, A_{k}\right\}$ and $A_{j}=\left\{x \mid x_{0}=j\right\}$. It transpires that if $\phi$ is a finitary isomorphism with finite expected code lengths between subshifts of finite type $\sigma$ and $\tau$, then there exists 
a finite valued measurable $g$ such that

$$
I_{\sigma}=I_{\tau} \circ \phi+g \circ \sigma-g
$$

i.e. $I_{\sigma}$ and $I_{\tau} \circ \phi$ are cohomologous. (A coboundary is a function of the form $g \circ \sigma-g$.) One way of extracting an invariant from this equality is to define the information variance

$$
\sigma^{2}(\sigma)=\lim _{n \rightarrow+\infty} \frac{1}{n} \int\left(I_{\sigma}+I_{\sigma} \circ \sigma+\cdots+I_{\sigma} \circ \sigma^{n-1}-n h(\sigma)\right)^{2} d m
$$

if this exists. Thus if $\phi$ is a finitary isomorphism with finite expected code lengths between Markov shifts $\sigma, \tau$ then $\sigma^{2}(\sigma)=\sigma^{2}(\tau)$. It is a simple matter to produce examples with $h(\sigma)=h(\tau)$ but $\sigma^{2}(\sigma) \neq \sigma^{2}(\tau)$. This idea was first introduced in [64] in connection with the notion of regular isomorphism.

3.4. The $\beta$-function and natural invariants. As mentioned above any measurepreserving isomorphism $\phi$ between two subshifts of finite type takes the form $\phi=\left(\phi_{0} \circ\right.$ $\left.\sigma^{n}\right)$, where $\phi_{0}$ is a map from the first shift space to the symbol space of the second space. To say that an isomorphism $\phi$ between two such subshifts $\sigma, \tau$ is regular amounts to saying that $\phi_{0}$ has bounded anticipation (but perhaps infinite memory) and that the corresponding statement holds for $\phi^{-1}$.

Selim Tuncel introduced the so-called $\beta$-function which for a Markov shift $\sigma_{P}$ equals the exponential of the pressure of the function $t \log P\left(x_{0}, x_{1}\right)$, that is, $\beta_{P}(t)$ is the maximum eigenvalue of $P^{t}$ (where $P^{t}(i, j)=(P(i, j))^{t}$ ) and showed that when $\sigma_{P}, \sigma_{Q}$ are regularly isomorphic, $\beta_{P}=\beta_{Q}[\mathbf{1 2 3}]$. A key fact in the proof is the boundedness of the cobounding function $g$. However, there is no guarantee that $g$ is bounded when $\sigma_{P}, \sigma_{Q}$ are finitary isomorphic with finite expected code lengths. Nevertheless, Parry and Schmidt [64] showed that (modulo a null set) $g$ assumes a countable number of values, which facilitates the introduction of other invariants $\Gamma_{P}, \Delta_{P}$ and $c_{P} \Delta_{P}$ (to be defined shortly). In a subsequent paper [111], Klaus Schmidt went on to show that a finitary isomorphism with finite expected code lengths guarantees $\beta_{P}=\beta_{Q}$. In this connection, one should note that the invariant $\beta_{P}$ contains topological entropy, measure-theoretic entropy and information variance.

Wolfgang Krieger defined an invariant $\Delta_{P}$ of finitary isomorphism with finite expected code length which gave an alternative method for producing counter-examples [124]. The group $\Delta_{P}$ is defined as

$$
\Delta_{P}=\left\{\frac{P\left(i_{0}, i_{1}\right) \cdots P\left(i_{n-1}, i_{0}\right)}{P\left(i_{0}, j_{1}\right) \cdots P\left(j_{n-1}, i_{0}\right)}\right\}
$$

in other words $\Delta_{P}$ consists of ratios of (non-vanishing) weights of equal length cycles beginning and ending in the same state. This is a readily computable group. This was extended in [64] to the finitely generated group $\Gamma_{P}$ defined as the multiplicative group generated by all weights $P\left(i_{0}, i_{1}\right) \cdots P\left(i_{n-1}, i_{0}\right) \neq 0$. When $P$ is aperiodic it was shown that $\Gamma_{P} / \Delta_{P}$ is cyclic with canonical generator $c_{P} \Delta_{P}$ and that there exists a positive vector $r$ with $P(i, j) r_{j} / r_{i} \in \Gamma_{P}$ and $P(i, j) r_{j} / c_{P} r_{i} \in \Delta_{P}$. In particular, $\Gamma_{P}, \Delta_{P}, c_{P} \Delta_{P}$ and $\beta_{P}$ are all invariants of finitary isomorphism. 
The question of whether $\beta_{P}, \Delta_{P}$ and $c_{P} \Delta_{P}$ form a complete set of invariants remains open.

3.5. Flow equivalence. One can also consider flows that are suspensions of subshifts of finite type for which the periodic points are dense and there are uncountably many dense orbits. Given a subshift of finite type $\sigma: \Sigma_{A} \rightarrow \Sigma_{A}$, one can consider the suspension flow $\sigma_{t}: \Sigma_{A}^{1} \rightarrow \Sigma_{A}^{1}$ defined on the space

$$
\Sigma_{A}^{1}=\left\{(x, u) \in \Sigma_{A} \times[0,1] \mid(x, 1) \sim(\sigma x, 0)\right\}
$$

where $\sigma_{t}(x, u)=(x, u+t)$, subject to the identification.

Two matrices $A$ and $B$ are flow equivalent if there is a homeomorphism between $\Sigma_{A}^{1}$ and $\Sigma_{B}^{1}$ carrying flow lines to flow lines with the proper direction. In 1975, Parry and Sullivan showed that if $A$ and $B$ are flow equivalent then $\operatorname{det}(1-A)=\operatorname{det}(1-B)$ [42]. Bowen and Franks showed that the rings $\mathbb{Z}^{n} /\left(I-A^{n}\right) \mathbb{Z}^{n}$ are invariants for flow equivalence and, in 1984, Franks showed that together the Bowen-Franks invariant [100] and (the sign of) the Parry-Sullivan $\operatorname{det}(I-A)$ invariant form a complete invariant for flow equivalence [101].

\section{The later years}

4.1. Zeta functions and closed orbits. In 1983, Bill Parry returned to a favourite theme, the connection between number theory and ergodic theory. Motivated by an undergraduate lecture course he had given on the proof of the prime number theorem (i.e. that the number of primes less than $T$ was asymptotic to $T / \log T$ ) he considered the analogous result for certain suspended flows [61]. More precisely, given a subshift of finite type $\sigma: \Sigma_{A} \rightarrow \Sigma_{A}$ and a continuous positive function $r: \Sigma_{A} \rightarrow \mathbb{R}$ one defines

$$
\Sigma_{A}^{r}=\left\{(x, u) \in \Sigma_{A} \times \mathbb{R} \mid 0 \leq u \leq r(x)\right\} / \sim
$$

with the identification $(x, r(x)) \sim(\sigma x, 0)$. The suspended flow $\sigma_{t}^{r}: \Sigma_{A}^{r} \rightarrow \Sigma_{A}^{r}$ is defined by $\sigma_{t}^{r}(x, u)=(x, u+t)$, subject to the identification. The closed orbits $\tau$ for this flow correspond to periodic orbits $\sigma^{n} x=x$ for the shift $\sigma$, with period $\lambda(\tau)=r^{n}(x):=$ $r(x)+r(\sigma x)+\cdots+r\left(\sigma^{n-1} x\right)$. The flow is topologically weak mixing if the lengths are not all integer multiples of a constant.

The analogue with prime numbers comes from the countable set of (prime) closed orbits with weights $N(\tau)=e^{h \lambda(\tau)}$, where $h>0$ is the topological entropy of the flow. In the case of locally constant functions $r: \Sigma_{A} \rightarrow \mathbb{R}$, Bill Parry showed that for a weak mixing suspension by a locally constant function the number $\pi(t)$ of orbits $\tau$ with $N(\tau) \leq T$ satisfies

$$
\pi(T) \sim \frac{T}{\log T} \quad \text { as } T \rightarrow+\infty
$$

(i.e. $\lim _{T \rightarrow+\infty} \pi(T) /(T / \log T)=1$ ) [64]. This was subsequently extended to Hölder continuous functions $r: \Sigma_{A} \rightarrow \mathbb{R}$ by Parry and Pollicott and thus, through the work of Bowen on modelling hyperbolic flows by suspended flows, applied to weak mixing hyperbolic flows (including Axiom A flows on basic sets and, in particular, geodesic flows on negatively curved manifolds) [65]. Similar results had been proved by Margulis for 
Anosov flows, using a very different approach, and at the time his work was not widely known in the West [105].

The basic approach was to study a dynamical zeta function introduced by Ruelle [125],

$$
\zeta(s)=\prod_{\tau}\left(1-e^{-s h \lambda(\tau)}\right)^{-1} .
$$

The key idea was to establish results on its domain analogous to those which hold for the Riemann zeta function and are used in the proof of the prime number theorem. In the case of locally constant functions $f(x)=f\left(x_{0}, x_{1}\right)$ one can associate a family of matrices $P^{s}$ $(s \in \mathbb{C})$ defined by $P^{s}(i, j)=A(i, j) e^{-s r(i, j)}$ and then $\zeta(s)=\operatorname{det}\left(I-P^{s}\right)^{-1}$. For general Hölder continuous functions the analysis of the zeta function $\zeta(s)$ is more complicated with Ruelle transfer operators replacing the matrices $P^{s}$.

Parry and Pollicott continued this analogy by establishing a dynamical analogue of Chebatorev's theorem from number theory, describing the equidistribution of closed orbits for hyperbolic flows according to how they lift to finite covers [66]. They were collaborating on another dynamical analogue of a number theoretic result, Bauer's theorem, at the time of Parry's death [94].

Bill Parry also used the zeta function approach to develop an alternative proof of Bowen's well-known result on the equidistribution of closed orbits for an Axiom A flow $\phi_{t}[\mathbf{6 5}, \mathbf{9 8}]$. More precisely, if $\mu$ denotes the measure of maximal entropy for the flow then for any continuous function $g$ and $\epsilon>0$,

$$
\frac{\sum_{T \leq \lambda(\tau) \leq T+\epsilon} \int_{0}^{\lambda(\tau)} g\left(\phi_{t} x_{\tau}\right) d t}{\sum_{T \leq \lambda(\tau) \leq T+\epsilon} \lambda(\tau)} \rightarrow \int g d \mu \quad \text { as } T \rightarrow+\infty,
$$

where $x_{\tau} \in \tau$. He subsequently showed that if $k$ is a Hölder continuous function and $\lambda_{k}(\tau)=\int_{0}^{\lambda(\tau)} k\left(\phi_{t} x_{\tau}\right) d t$ then for any continuous function $g$ and $\epsilon>0$,

$$
\frac{\sum_{T \leq \lambda(\tau) \leq T+\epsilon} \int_{0}^{\lambda(\tau)} g\left(\phi_{t} x_{\tau}\right) e^{\lambda_{k}(\tau)} d t}{\sum_{T \leq \lambda(\tau) \leq T+\epsilon} \lambda(\tau) e^{\lambda_{k}(\tau)}} \rightarrow \int g d \mu_{k} \quad \text { as } T \rightarrow+\infty,
$$

where $\mu_{k}$ denotes the unique equilibrium state associated to $k$, i.e. the unique invariant probability measure for which $h(\mu)+\int k d \mu$ is maximized [72].

4.2. Skew products and mixing. Given a hyperbolic diffeomorphism $\phi: X \rightarrow X$, a compact group Lie group $G$ and a Hölder continuous function $f: X \rightarrow G$, we can consider a skew product $\widehat{\phi}: X \times G \rightarrow X \times G$ defined by $\widehat{\phi}(x, g)=(\phi x, f(x) g)$. Let $\mu$ be an equilibrium state (for a Hölder continuous function) and let $\lambda$ be the normalized Haar measure on $G$, then $\widehat{\mu}=\mu \times \lambda$ is a $\widehat{\phi}$-invariant measure. There is a well-known criterion for ergodicity of $\widehat{\phi}$ with respect to $\widehat{\mu}$ due to Harvey Keynes and Dan Newton, namely, for any unitary representation $R: G \rightarrow U(n)$, the equation $R(f(x)) u(x)=u(\phi x)$ has only trivial solutions.

In the case that $G=\mathbb{T}^{d}$, Parry and Pollicott considered the genericity of functions $f: X \rightarrow G$ for which the associated skew product is ergodic (or mixing) [87]. Subsequently, Mike Field and Bill Parry extended these results to the case of general compact Lie groups $G$ [90]. 
4.3. Liv̌sic's theorem and cocycles. Let $\phi: X \rightarrow X$ be a mixing hyperbolic diffeomorphism (or subshift of finite type). A. N. Liv̌sic's original theorems gave criteria for a Hölder continuous function $f: X \rightarrow \mathbb{R}$ (or $f: X \rightarrow K$, where $K$ is a compact abelian group) to be a coboundary [126]. More precisely, assume $f: X \rightarrow \mathbb{R}$ so that whenever $\phi^{n} x=x$ we have that $\sum_{i=0}^{n-1} f\left(\phi^{i} x\right)=0$ and then there exists a Hölder continuous $u$ for which $f=u \circ \phi-u$. Bill Parry showed that a version of Liv̌sic's theorem for periodic points (or homoclinic points) holds for finite non-abelian groups [91]. An alternative proof was given by Schmidt [112].

In the context of higher rank abelian actions, Bill Parry published two related papers on the triviality of cocycles $[\mathbf{8 0}, \mathbf{8 4}]$.

4.4. Unfinished work. At the time of his death, Bill Parry was still working on several projects. This included an analogue of Bauer's theorem from number theory for skew products (with Mark Pollicott) [94] and work on Shannon entropy (with Doureid Hamdan and Jean-Paul Thouvenot) [95]. Both articles appear in this volume.

Of the many other questions Bill was working on, one related to aperiodic subshifts of finite type, continuous functions $f: X_{A} \rightarrow G$ into finite abelian groups, and their associated zeta functions

$$
\zeta(z)=\exp \left(\sum_{n=1}^{\infty} \frac{z^{n}}{n} \sum_{\sigma^{n} x=x} g(x) g(\sigma x) \cdots g\left(\sigma^{n-1} x\right)\right) .
$$

The zeta function will clearly be the same for functions which differ by coboundaries or which are related by automorphisms.

Question. Are there essentially only a finite number of inequivalent such $f$ which give rise to the same zeta function?

He left hand-written notes on this problem, including several carefully worked out examples.

\section{PhD students of William Parry}

D. Newton (PhD, Birmingham, 1966)

M. Haque (DPhil, Sussex, 1967)

P. Walters (DPhil, Sussex, 1967)

S. Rudolfer (PhD, Imperial, 1968)

R. Thomas (PhD, Warwick, 1969)

P. Humphries (PhD, Warwick, 1971)

A. Mohamed (PhD, Warwick, 1975)

R. Felgett (PhD, Warwick, 1976)

S. M. Rees (PhD, Warwick, 1977)

M. R. Palmer (PhD, Warwick, 1979)

S. Tuncel (PhD, Warwick, 1981)

M. Pollicott (PhD, Warwick, 1984)

R. Nair (PhD, Warwick, 1986)

R. Cowen (PhD, Warwick, 1987) 
R. Sharp (PhD, Warwick, 1990)

S. Waddington (PhD, Warwick, 1992)

P. Araujo (PhD, Warwick, 1992)

M. S. M. Noorani (PhD, Warwick, 1993)

C. P. Walkden (PhD, Warwick, 1997)

L. Lambrou (PhD, Warwick, 1998)

\section{Professional activities and honours}

6.1. Editorships. Editor of Ergodic Theory and Dynamical Systems: 1981-2006 (as Executive Editor (1986-1996) and as Managing editor (1997-2001)); and Editor (Ergodic Theory) for the London Mathematical Society 1979-1983.

6.2. Addresses and honours. International Congress of Mathematicians, 1970; British Mathematical Colloquium, 1970 and 1985; Bowen lectures (Berkeley), 1985. Elected Fellow of the Royal Society of London, 1984.

\section{Books}

Bill Parry published four books. His first book Entropy and Generators in Ergodic Theory is a very clearly written specialist account of the subject at that time [26]. The book Topics in Ergodic Theory is an admirably concise introductory text based on lectures given at Warwick University [53].

The book Classification Problems in Ergodic Theory (with Selim Tuncel) describes the status of various classifications (of both subshifts of finite type and Markov chains) circa 1980 [58].

Finally, the book Zeta functions and the periodic orbit structure of hyperbolic dynamics (with Mark Pollicott) is again based on courses given at Warwick by the authors [74].

\section{REFERENCES}

\section{Papers authored by William Parry}

[1] W. Parry. On the $\beta$-expansions of real numbers. Acta Math. Acad. Sci. Hungar. 11 (1960), 401-416.

[2] W. Parry. Ergodic properties of some permutation processes. Biometrika 49 (1962), 151-154.

[3] S. Kakutani and W. Parry. Infinite measure preserving transformations with mixing. Bull. Amer. Math. Soc. 69 (1963), 752-756.

[4] W. Parry. An ergodic theorem of information theory without invariant measure. Proc. London Math. Soc. 13(3) (1963), 605-612.

[5] W. Parry. Intrinsic Markov chains. Trans. Amer. Math. Soc. 112 (1964), 55-66.

[6] W. Parry. On Rohlin's formula for entropy. Acta Math. Acad. Sci. Hungar. 15 (1964), 107-113.

[7] W. Parry. Note on the ergodic theorem of Hurewicz. J. London Math. Soc. 39 (1964), 202-210.

[8] W. Parry. Representations for real numbers. Acta Math. Acad. Sci. Hungar. 15 (1964), 95-105.

[9] F. Hahn and W. Parry. Minimal dynamical systems with quasi-discrete spectrum. J. London Math. Soc. 40 (1965), 309-323.

[10] W. Parry. Ergodic and spectral analysis of certain infinite measure preserving transformations. Proc. Amer. Math. Soc. 16 (1965), 960-966. 
[11] H. Hoare and W. Parry. Affine transformations with quasi-discrete spectrum. I. J. London Math. Soc. 41 (1966), 88-96.

[12] W. Parry. Generators and strong generators in ergodic theory. Bull. Amer. Math. Soc. 72 (1966), 294-296.

[13] H. Hoare and W. Parry. Affine transformations with quasi-discrete spectrum. II. J. London Math. Soc. 41 (1966), 529-530.

[14] W. Parry. Symbolic dynamics and transformations of the unit interval. Trans. Amer. Math. Soc. 122 (1966), 368-378.

[15] W. Parry. On the coincidence of three invariant $\sigma$-algebras associated with an affine transformation. Proc. Amer. Math. Soc. 17 (1966), 1297-1302.

[16] H. Hoare and W. Parry. Semi-groups of affine transformations. Quart. J. Math. Oxford Ser. (2) 17 (1966), 106-111.

[17] D. Newton and W. Parry. On a factor automorphism of a normal dynamical system. Ann. Math. Statist. 37 (1966), 1528-1533.

[18] R. Laxton and W. Parry. On the periodic points of certain automorphisms and a system of polynomial identities. J. Algebra 6 (1967), 388-393.

[19] W. Parry. Principal partitions and generators. Bull. Amer. Math. Soc. 73 (1967), 307-309.

[20] W. Parry. Generators for perfect partitions. Dokl. Akad. Nauk SSSR 173 (1967), 264-266.

[21] W. Parry. Aperiodic transformations and generators. J. London Math. Soc. 43 (1968), 191-194.

[22] F. Hahn and W. Parry. Some characteristic properties of dynamical systems with quasi-discrete spectra. Math. Systems Theory 2 (1968), 179-190.

[23] W. Parry. Zero entropy of distal and related transformations. Topological Dynamics (Symposium, Colorado State University, Fort Collins, CO, 1967). Eds. J. Auslander and W. H. Gottschalk. Benjamin, New York, 1968, pp. 383-389.

[24] W. Parry. Ergodic properties of affine transformations and flows on nilmanifolds. Amer. J. Math. 91 (1969), 757-771.

[25] W. Parry. Compact abelian group extensions of discrete dynamical systems. Z. Wahrsch. Verw. Gebiete 13 (1969), 95-113.

[26] W. Parry. Entropy and Generators in Ergodic Theory. W. A. Benjamin, New York, 1969.

[27] W. Parry. Spectral analysis of $G$-extensions of dynamical systems. Topology 9 (1970), 217-224.

[28] W. Parry. Dynamical systems on nilmanifolds. Bull. London Math. Soc. 2 (1970), 37-40.

[29] W. Parry and P. Walters. Minimal skew-product homeomorphisms and coalescence. Compos. Math. 22 (1970), 283-288.

[30] W. Parry. Metric classification of ergodic nilflows and unipotent affines. Amer. J. Math. 93 (1971), 819-828.

[31] W. Parry. Ergodic theory of $G$-spaces. Actes du Congrès International des Mathématiciens (Nice, 1970, Tome 2). Gauthier-Villars, Paris, 1971, pp. 921-924.

[32] W. Parry and P. Walters. Endomorphisms of a Lebesgue space. Bull. Amer. Math. Soc. 78 (1972), 272-276. Also: Erratum to 'Endomorphisms of a Lebesgue space'. Bull. Amer. Math. Soc. 78 (1972), 628.

[33] R. Azencott and W. Parry. Stability of group representations and Haar spectrum. Trans. Amer. Math. Soc. 172 (1972), 317-327.

[34] W. Parry. Cocycles and velocity changes. J. London Math. Soc. 5(2) (1972), 511-516.

[35] R. Jones and W. Parry. Compact abelian group extensions of dynamical systems. II. Compos. Math. 25 (1972), 135-147.

[36] W. Parry. Dynamical representations in nilmanifolds. Compos. Math. 26 (1973), 159-174.

[37] W. Parry. Notes on a posthumous paper by F. Hahn. Israel J. Math. 16 (1973), 38-45.

[38] W. Parry. Class properties of dynamical systems. Recent Advances in Topological Dynamics (Proc. Conf., Yale University, New Haven, CT, 1972; in honor of Gustav Arnold Hedlund) (Lecture Notes in Mathematics, 318). Springer, Berlin, 1973, pp. 218-225.

[39] W. Parry. A note on cocycles in ergodic theory. Compos. Math. 28 (1974), 343-350.

[40] R. Fellgett and W. Parry. Endomorphisms of a Lebesgue space. II. Bull. London Math. Soc. 7 (1975), 151-158.

[41] W. Parry. Endomorphisms of a Lebesgue space. III. Conference on Ergodic Theory and Topological Dynamics (Kibbutz Lavi, 1974). Israel J. Math. 21 (1975), 167-172.

[42] W. Parry and D. Sullivan. A topological invariant of flows on 1-dimensional spaces. Topology 14 (1975), 297-299. 
[43] W. Parry and K. Schmidt. A note on cocycles of unitary representations. Proc. Amer. Math. Soc. 55 (1976), 185-190.

[44] W. Parry. Some classification problems in ergodic theory. Sankhyā Ser. A 38 (1976), 38-43.

[45] W. Parry and R. Williams. Block coding and a zeta function for finite Markov chains. Proc. London Math. Soc. (3) 35 (1977), 483-495.

[46] W. Parry. A finitary classification of topological Markov chains and sofic systems. Bull. London Math. Soc. 9 (1977), 86-92.

[47] W. Parry. The information cocycle and $\varepsilon$-bounded codes. Israel J. Math. 29 (1978), 205-220.

[48] M. R. Palmer, W. Parry and P. Walters. Large sets of endomorphisms and of $g$-measures. The Structure of Attractors in Dynamical Systems (Proc. Conf., North Dakota State University, Fargo, ND, 1977) (Lecture Notes in Mathematics, 668). Eds. N. G. Markley, J. C. Martin and W. Perrizo. Springer, Berlin, 1978, pp. 191-210.

[49] H. Helson and W. Parry. Cocycles and spectra. Ark. Mat. 16(2) (1978), 195-206.

[50] W. Parry. An information obstruction to finite expected coding length. Ergodic Theory (Proc. Conf., Math. Forschungsinst., Oberwolfach, 1978) (Lecture Notes in Mathematics, 729). Springer, Berlin, 1979, pp. 163-168.

[51] W. Parry. Finitary isomorphisms with finite expected code lengths. Bull. London Math. Soc. 11 (1979), 170-176.

[52] W. Parry. The Lorenz attractor and a related population model. Ergodic Theory (Proc. Conf., Math. Forschungsinst., Oberwolfach, 1978) (Lecture Notes in Mathematics, 729). Eds. M. Denker and K. Jacobs. Springer, Berlin, 1979, pp. 169-187.

[53] W. Parry. Topics in Ergodic Theory (Cambridge Tracts in Mathematics, 75). Cambridge University Press, Cambridge, 1981.

[54] W. Parry. Finitary isomorphisms with finite expected code-lengths. II. J. London Math. Soc. (2) 24 (1981), 569-576.

[55] W. Parry. Self-generation of self-replicating maps of an interval. Ergod. Th. \& Dynam. Sys. 1 (1981), 197-208.

[56] W. Parry and S. Tuncel. On the classification of Markov chains by finite equivalence. Ergod. Th. \& Dynam. Sys. 1 (1981), 303-335.

[57] W. Parry. The classification of topological Markov chains: adapted shift equivalence. Israel J. Math. 38 (1981), 335-344.

[58] W. Parry and S. Tuncel. Classification Problems in Ergodic Theory (London Mathematical Society Lecture Note Series, 67). Cambridge University Press, Cambridge, 1982.

[59] W. Parry and S. Tuncel. On the stochastic and topological structure of Markov chains. Bull. London Math. Soc. 14 (1982), 16-27.

[60] W. Parry and S. Tuncel. Two classification problems for finite state Markov chains. Ergodic Theory and Related Topics (Proc. Conf., Vitte, 1981) (Mathematical Research, 12). Ed. H. Michel. AkademieVerlag, Berlin, 1982, pp. 153-159.

[61] W. Parry. An analogue of the prime number theorem for closed orbits of shifts of finite type and their suspensions. Israel J. Math. 45 (1983), 41-52.

[62] W. Parry and M. Pollicott. An analogue of the prime number theorem for closed orbits of Axiom A flows. Ann. of Math. (2) 118 (1983), 573-591.

[63] W. Parry and K. Schmidt. Invariants of finitary isomorphisms with finite expected code-lengths. Conference in Modern Analysis and Probability (New Haven, CT, 1982) (Contemporary Mathematics, 26). Eds. R. Beals, A. Beck, A. Bellow and A. Hajian. American Mathematical Society, Providence, RI, 1984, pp. 301-307.

[64] W. Parry and K. Schmidt. Natural coefficients and invariants for Markov-shifts. Invent. Math. 76 (1984), 15-32.

[65] W. Parry. Bowen's equidistribution theory and the Dirichlet density theorem. Ergod. Th. \& Dynam. Sys. 4 (1984), 117-134.

[66] W. Parry and M. Pollicott. The Chebotarov theorem for Galois coverings of Axiom A flows. Ergod. Th. \& Dynam. Sys. 6 (1986), 133-148.

[67] W. Parry. Synchronisation of canonical measures for hyperbolic attractors. Comm. Math. Phys. 106 (1986), 267-275.

[68] J. C. Alexander and W. Parry. Discerning fat baker's transformations. Dynamical Systems (College Park, MD, 1986-87) (Lecture Notes in Mathematics, 1342). Springer, Berlin, 1988, pp. 1-6. 
[69] W. Parry. Equilibrium states and weighted uniform distribution of closed orbits. Dynamical Systems (College Park, MD, 1986-87) (Lecture Notes in Mathematics, 1342). Springer, Berlin, 1988, pp. 617-625.

[70] W. Parry. Problems and perspectives in the theory of Markov shifts. Dynamical Systems (College Park, MD, 1986-87) (Lecture Notes in Mathematics, 1342). Springer, Berlin, 1988, pp. 626-637.

[71] W. Parry. Decoding with two independent processes. Measure and Measurable Dynamics (Rochester, NY, 1987) (Contemporary Mathematics, 94). Eds. R. D. Mauldin, R. M. Shortt and C. E. Silva. American Mathematical Society, Providence, RI, 1989, pp. 207-209.

[72] W. Parry. Temporal and spatial distribution of closed orbits of hyperbolic dynamical systems. Measure and Measurable Dynamics (Rochester, NY, 1987) (Contemporary Mathematics, 94). Eds. R. D. Mauldin, R. M. Shortt and C. E. Silva. American Mathematical Society, Providence, RI, 1989, pp. 211-216.

[73] Z. Coelho and W. Parry. Central limit asymptotics for shifts of finite type. Israel J. Math. 69 (1990), 235-249.

[74] W. Parry and M. Pollicott. Zeta functions and the periodic orbit structure of hyperbolic dynamics. Astérisque 187-188 (1990), 1-268.

[75] W. Parry. Notes on coding problems for finite state processes. Bull. London Math. Soc. 23 (1991), 1-33.

[76] W. Parry. A cocycle equation for shifts. Symbolic Dynamics and its Applications (New Haven, CT, 1991) (Contemporary Mathematics, 135). Ed. P. Walters. American Mathematical Society, Providence, RI, 1992, pp. 327-333.

[77] W. Parry. In general a degree two map is an automorphism. Symbolic Dynamics and its Applications (New Haven, CT, 1991) (Contemporary Mathematics, 135). Ed. P. Walters. American Mathematical Society, Providence, RI, 1992, pp. 335-338.

[78] M. S. M. Noorani and W. Parry. A Chebotarev theorem for finite homogeneous extensions of shifts. Bol. Soc. Brasil. Mat. (N.S.) 23 (1992), 137-151.

[79] W. Parry. Remarks on Williams' problem. Differential Equations, Dynamical Systems, and Control Science (Lecture Notes in Pure and Applied Mathematics, 152). Eds. K. D. Elworthy, W. N. Everitt and E. B. Lee. Dekker, New York, 1994, pp. 235-246.

[80] W. Parry. Instances of cohomological triviality and rigidity. Ergod. Th. \& Dynam. Sys. 15 (1995), 685-696.

[81] W. Parry. Ergodic properties of a one-parameter family of skew-products. Nonlinearity 8 (1995), 821-825.

[82] W. Parry. Automorphisms of the Bernoulli endomorphism and a class of skew-products. Ergod. Th. \& Dynam. Sys. 16 (1996), 519-529.

[83] W. Parry. Squaring and cubing the circle-Rudolph's theorem. Ergodic Theory of $\mathbb{Z}^{d}$ Actions (Warwick, 1993-1994) (London Mathematical Society Lecture Note Series, 228). Eds. M. Pollicott and K. Schmidt. Cambridge University Press, Cambridge, 1996, pp. 177-183.

[84] W. Parry. Cohomology of permutative cellular automata. Israel J. Math. 99 (1997), 315-333.

[85] W. Parry. Skew products of shifts with a compact Lie group. J. London Math. Soc. (2) 56 (1997), 395-404.

[86] W. Parry and M. Pollicott. The Livšic cocycle equation for compact Lie group extensions of hyperbolic systems. J. London Math. Soc. (2) 56 (1997), 405-416.

[87] W. Parry and M. Pollicott. Stability of mixing for toral extensions of hyperbolic systems. Proc. Steklov Inst. Math. 216 (1997), 350-359.

[88] Z. Coelho, W. Parry and R. Williams. A note on Livšic's periodic point theorem. Topological Dynamics and Applications (Contemporary Mathematics, 215). American Mathematical Society, Providence, RI, 1998, pp. 223-230.

[89] Z. Coelho and W. Parry. Shift endomorphisms and compact Lie extensions. Bol. Soc. Brasil. Mat. (N.S.) 29 (1998), 163-179.

[90] M. Field and W. Parry. Stable ergodicity of skew extensions by compact Lie groups. Topology 38 (1999), 167-187.

[91] W. Parry. The Livšic periodic point theorem for non-abelian cocycles. Ergod. Th. \& Dynam. Sys. 19 (1999), 687-701.

[92] Z. Coelho and W. Parry. Ergodicity of $p$-adic multiplications and the distribution of Fibonacci numbers. Topology, Ergodic Theory, Real Algebraic Geometry (American Mathematical Society Translation Series 2, 202). Eds. V. Turaev and A. Vershik. American Mathematical Society, Providence, RI, 2001, pp. 51-70. 
[93] W. Parry and M. Pollicott. Skew products and Liv̌sic theory. Representation Theory, Dynamical Systems, and Asymptotic Combinatorics (American Mathematical Society Translation Series 2, 217). Eds. V. Kaimanovich and A. Lodkin. American Mathematical Society, Providence, RI, 2006, pp. 139-165.

[94] W. Parry and M. Pollicott. An analogue of Bauer's theorem for closed orbits of skew products. Ergod. Th. \& Dynam. Sys. 28 (2008), 535-546.

[95] D. Hamdan, W. Parry and J.-P. Thouvenot. Shannon entropy for stationary processes and dynamical systems. Ergod. Th. \& Dynam. Sys. 28 (2008), 447-480.

[96] W. Parry. An elementary construction of $C^{r}$ renormalizing maps, volume in honour of Zeeman's 60th Birthday, unpublished.

\section{Other articles cited in the survey}

[97] J. Ashley. Bounded-to-1 factors of an aperiodic shift of finite type are 1-to-1 almost everywhere factors also. Ergod. Th. \& Dynam. Sys. 10 (1990), 615-625.

[98] R. Bowen. The equidistribution of closed geodesics. Amer. J. Math. 94 (1972), 413-423.

[99] R. Bowen. Equilibrium States and the Ergodic Theory of Anosov Diffeomorphisms (Lecture Notes in Mathematics, 470). Springer, Berlin, 1975.

[100] R. Bowen and J. Franks. Homology for zero-dimensional nonwandering sets. Ann. of Math. (2) 106 (1977), 73-92.

[101] J. Franks. Flow equivalence of subshifts of finite type. Ergod. Th. \& Dynam. Sys. 4 (1984), 53-66.

[102] N. A. Friedman and D. S. Ornstein. On isomorphism of weak Bernoulli transformations. Adv. Math. 5 (1970), 365-394.

[103] K. H. Kim and F. W. Roush. The Williams conjecture is false for irreducible subshifts. Ann. of Math. (2) 149 (1999), 545-558.

[104] B. Marcus and S. Tuncel. Matrices of polynomials, positivity, and finite equivalence of Markov chains. J. Amer. Math. Soc. 6 (1993), 131-147.

[105] G. A. Margulis. On Some Aspects of the Theory of Anosov Systems (With a Survey by Richard Sharp: Periodic Orbits of Hyperbolic Flows). Springer, Berlin, 2004.

[106] D. W. Morris. Ratner's Theorems on Unipotent Flows (Chicago Lectures in Mathematics). University of Chicago Press, Chicago, IL, 2005.

[107] A. Nogueira. The three-dimensional Poincaré continued fraction algorithm. Israel J. Math. 90(1-3) (1995), 373-401.

[108] D. Ornstein. Bernoulli shifts with the same entropy are isomorphic. Adv. Math. 4 (1970), 337-352.

[109] V. A. Rohlin. Lectures on the entropy theory of transformations with invariant measure. Uspehi Mat. Nauk 22 (1967), 3-56 (in Russian).

[110] D. Ruelle. A variational formulation of equilibrium statistical mechanics and the Gibbs phase rule. Comm. Math. Phys. 5 (1967), 324-329.

[111] K. Schmidt. Invariants for finitary isomorphisms with finite expected code lengths. Invent. Math. 76 (1984), 33-40.

[112] K. Schmidt. Remarks on Livšic' theory for nonabelian cocycles. Ergod. Th. \& Dynam. Sys. 19(3) (1999), $703-721$.

[113] F. Schweiger. Ergodic Theory of Fibred Systems and Metric Number Theory (Oxford Science Publications). The Clarendon Press, Oxford University Press, New York, 1995.

[114] M. Shub and D. Sullivan. Expanding endomorphisms of the circle revisited. Ergod. Th. \& Dynam. Sys. 5 (1985), 285-289.

[115] S. Smale. Differentiable dynamical systems. Bull. Amer. Math. Soc. 73 (1967), 747-817.

[116] P. Walters. A variational principle for the pressure of continuous transformations. Amer. J. Math. 97 (1975), 937-971.

[117] R. Williams. Classification of subshifts of finite type. Ann. of Math. (2) 98 (1973), 120-153. Also: Erratum to 'Classification of subshifts of finite type'. Ann. of Math. (2) 99 (1974), 380-381.

[118] A. Rényi. Representations for real numbers and their ergodic properties. Acta Math. Acad. Sci. Hungar. 8 (1957), 477-493.

[119] V. A. Rohlin. Generators in ergodic theory II. Vestnik Leningrad. Univ. 20 (1965), 68-72.

[120] R. Adler and B. Weiss. Similarity of Automorphisms of the Torus (Memoirs of the American Mathematical Society, 98). American Mathematical Society, Providence, RI, 1970. 
[121] R. Adler and B. Marcus. Topological Entropy and Equivalence of Dynamical Systems (Memoirs of the American Mathematical Society, 219). American Mathematical Society, Providence, RI, 1979.

[122] M. Keane and M. Smorodinsky. Bernoulli schemes of the same entropy are finitarily isomorphic. Ann. of Math. 109 (1979), 397-406.

[123] S. Tuncel. Conditional pressure and coding. Israel J. Math. 39 (1981), 101-112.

[124] W. Krieger. On the finitary isomorphisms of Markov shifts that have finite expected coding time. Z. Wahrsch. Verw. Gebiete 65 (1983), 323-328.

[125] D. Ruelle. Zeta-functions for expanding maps and Anosov flows. Invent. Math. 34 (1976), 231-242.

[126] A. N. Livšic. Certain properties of the homology of $Y$-systems. Math. Notes 10 (1971), 758-763.

\section{СамвRIDGE JUURNALS}

